\title{
Stick-Slip Transition at the Nanometer Scale
}

\author{
Christophe Cheikh and Ger Koper \\ DelftChemTech, Delft University of Technology, Julianalaan 136, 2628 BL Delft, The Netherlands
}

(Received 7 May 2003; published 8 October 2003)

\begin{abstract}
We report the first observation of a stick-slip transition of surfactant solution flow through nanopores. From the experimental data, we were able to determine both the slip length and the critical wall shear stress from which slip occurs. Whereas the latter is found to increase linearly with the concentration, the former remains constant and approximately equal to $20 \mathrm{~nm}$ over the studied range of concentrations. We model slip to occur in the surfactant bilayer adsorbed at the nanopore wall. The stick-slip transition is then related to a reorganization of the surfactant bilayer from an entangled structure into independent layers flowing past one another, as evidenced by independent surface plasmon resonance experiments. We conclude from our analysis that surfactant solutions are always slipping in larger tubes. However, the larger the tube diameter, the smaller the relative slip contribution to the total flow.
\end{abstract}

Standard textbooks assume a stick boundary condition at the wall when calculating fluid flow through cylindrical pipes, even though it is usually noted that there is no solid physical basis for this assumption (e.g., [1]). Systematic experiments showed that in both cases of hydrophobic fluid flow through hydrophilic capillaries and water flow through hydrophobic capillaries slippage does occur [2]. These experiments were interpreted in terms of an apparent increase of the capillary radius called the "slip length." Recent surface force experiments indicate that also on the molecular level slippage along surfaces does occur (see, e.g., review by [3]). For instance, Bonaccurso [4] observed slippage of water over hydrophilic surfaces and characterized it by a slip length equal to $8 \mathrm{~nm}$. In fact, a transition from stick to slip for surfactant solution flow along surfaces has been observed and the associated "yield stress" has been found to be of the order of $1 \mathrm{Nm}^{-2}$ [5,6]. Timmerman et al. [7] have brought the slippage of surfactant solutions along walls in connection with the washing power of detergents by associating it with the transfer of dirt particles from surfaces into solution. This would explain the apparent increase in washing power with decreasing dynamic surface tension $[8,9]$.

To describe mass transfer along the surface, the NavierStokes equation for incompressible Newtonian fluid flow in a capillary of radius $R$ is solved using the generalized boundary condition [10]

$$
\left.(\overrightarrow{\overrightarrow{1}}-\hat{n} \hat{n}) \cdot(\eta \vec{v}-2 \lambda \operatorname{sym}[\overrightarrow{\eta \nabla} \vec{v}] \cdot \hat{n})\right|_{r=R}=0,
$$

where $\hat{n}$ is the normal unit vector to the surface. Equation (1) states that, just like Stokes's friction law, the boundary fluid velocity is proportional to the tangential component of the shear stress at the boundary. The solution reads

$$
v(r)=-\frac{1}{4 \eta} P\left(r^{2}-2 \lambda R-R^{2}\right),
$$

with $P$ the pressure gradient in the capillary. The proportionality factor $\lambda$ in Eq. (1) is the slip length. Indeed, for $R \gg \lambda$, the extrapolated velocity vanishes at $r=R+\lambda$, see, e.g., [4,11]. In order to obtain a stick-slip transition as observed experimentally $[5,6,12,13]$ two regimes are identified, one at low shear stresses where there is no slip, i.e., $\lambda=0$, and another one at higher shear stress values where $\lambda$ is finite. To our knowledge, stick-slip transition has been observed only by surface force apparatus. In this Letter, we shall demonstrate the occurrence of such a transition for surfactant solution flow through nanopores.

Within experimentally accessible pressure gradients, the stick-slip transition is expected to be observable for small diameter capillaries only. In order to obtain a measurable flow many capillaries would have to be put in parallel, which is the case in a laser-etched polymer membrane. Such membranes are commercially available from Millipore (Massachusetts, USA) as Isopore VCTP and have a pore diameter of $0.1 \mu \mathrm{m}$, a thickness of $10 \mu \mathrm{m}$, and a porosity of $4 \%\left(5 \times 10^{12}\right.$ pores $\left./ \mathrm{m}^{2}\right)$. Because of the laser etching procedure the pores are straight and cylindrical. The pore size distribution is found by electron microscopy to be very narrow. The material is poly(carbonate), which renders strength to the membrane, coated by poly(vinyl pyrrolidone) (PVP) to make the surface hydrophilic. The membranes are guaranteed by the manufacturer up to 2 bars without deformation. We have determined the pore size distribution by means of electron microscopy and the coefficient of variation in the pore size is found to be of $1 \%$.

Accurate flow rates are imposed by a syringe pump Postnova PN1610 (Salt Lake City, USA) in the pressure range of 0 to 20 bars. The pressure drop across the membrane is measured by a Bronckhorst (Ruurlo, The Netherlands) pressure transmitter P-506C-FAC-22V connected to a digital computer. The accuracy of this device is $\pm 0.5 \%$. Pressure readings are averaged over 100 values 
(measured each $5 \mathrm{~s}$ ) and showed deviations less than $1 \%$. Pressure gradients are expressed in terms of measured pressure over actual membrane thickness. Surfactant solutions were prepared from crystalline sodium dodecyl sulphate (SDS), commercially available from Fisher Scientific (Loughborough, U.K.), dissolved in doubly distilled water. Concentrations are given in terms of the critical micelle concentration (CMC) which is $2.3 \mathrm{~g} / 1$ for the present surfactant. In a typical experiment, the membrane is first flushed with doubly distilled water at high flow rates. Then, a surfactant solution is flushed through the membrane stepping from low pressure values to high values and back again. Discrepancies between forward and backward curves are found to disappear after allowing for sufficient equilibration time. Fouling of the membrane is largely avoided by recycling surfactant solutions.

In Fig. 1, flow curves for some surfactant concentrations are presented. What is immediately clear from this figure is that the bottom two graphs show partially linear broken flow curves. It appears that beyond a certain
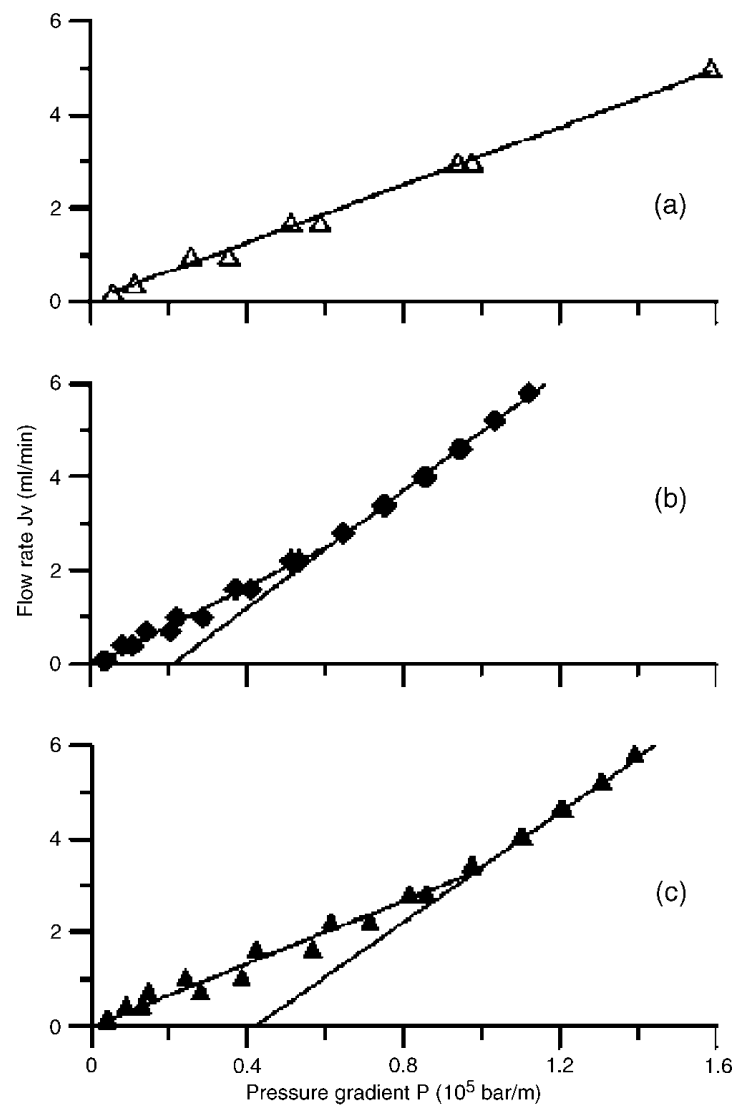

FIG. 1. Flow rate of surfactant solutions through a nanopore membrane versus applied pressure gradient for some SDS concentrations: $2 \times$ (top graph), $5 \times$ (middle graph), and $7 \times$ (bottom graph) the critical micellar concentration $(\mathrm{CMC}=$ $2.3 \mathrm{~g} / 1)$. The drawn lines are piecewise linear fit according to Eq. (2). Error bars are of the size of the symbols. critical pressure gradient, that tends to increase with surfactant concentration, the flow through the pores increases. This observation cannot be explained by an electroviscous effect since its consequence is to reduce monotonically the flow rate. As such, this effect does not predict a breakup [14].

Analogous experiments were conducted at higher and lower shear rate regimes by varying the pore size radius $(0.05$ and $0.22 \mu \mathrm{m})$. These results show a nonbroken relationship between flow rate and pressure gradient because the critical pressure gradient is beyond the experimentally accessible range.

We analyze these results in terms of stick-and-slip boundary conditions. Below the critical shear stress the flow appears to follow Poiseuille's law, i.e., the solution of Navier-Stokes's equation with stick boundary condition. The part above the critical pressure gradient can be described using a variation of Eq. (2) obtained using the boundary condition, Eq. (1), with an additional term for the critical shear stress. The fluid flow in that case can be expressed as

$$
J=\frac{\pi R^{4}}{8 \eta} N\left[P+\frac{4 \lambda}{R}\left(P-P_{e}\right) \theta\left(P-P_{e}\right)\right],
$$

with $\theta$ the Heaviside function and $N$ representing the number of pores in the membrane. This equation clearly shows the two contributions to slipping flow. The first term has the pure Poiseuille character and the corresponding fluid velocity profile is curved according to the ratio of pressure gradient over viscosity as in Eq. (2). The second contribution is due to slippage along the wall with a flat velocity profile as in plug flow. This equation is fit to the flow data using the following simple procedure. The data points for low pressure gradients give a linear regression which yields information on the number of pores in the membrane, the pore radius, and the viscosity. Likewise, the data points for high pressure gradients yield information on the critical pressure gradient and the slip length.

In the top graph of Fig. 2, one observes that the slip length hardly varies with surfactant concentration and remains of the order of $20 \mathrm{~nm}$. The bottom graph shows an almost linear dependence of the critical wall shear stress $\left(\sigma_{c}=\left.\eta \nabla v\right|_{r=R, P=P_{c}}\right)$ with concentration. Values for the slip length and the critical shear stress below surfactant concentration of $2 \mathrm{CMC}$ cannot be obtained from the flow rate versus pressure graph.

In the present situation, a bilayer of surfactant is formed where the polar head groups of the SDS molecules constituting the outer layer are directed towards the hydrophilic PVP surface and towards the aqueous phase $[15,16]$.

The results (Fig. 2) can be interpreted in terms of the properties of the adsorbed bilayer of surfactant in the same way as Yoshizawa et al. $[12,13]$ and Richetti et al. 


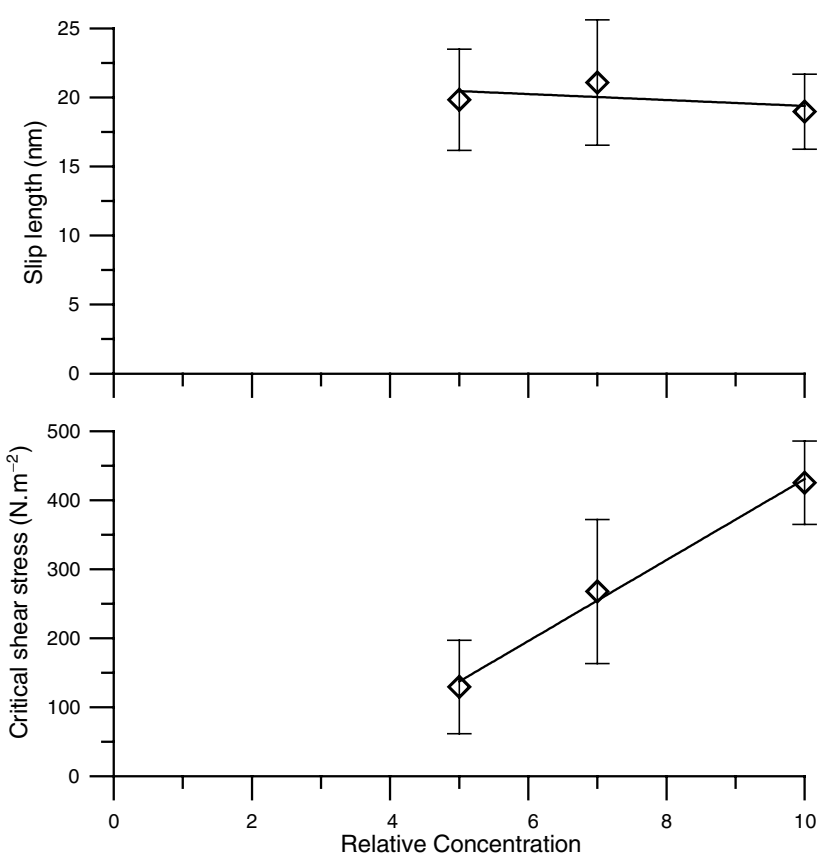

FIG. 2. Slip length (top) and critical shear stress (bottom) for various surfactant concentrations relative to the critical micellar micellar concentration $(\mathrm{cmc})$ of SDS. The drawn lines are linear fit to the data points.

[17] who both found such transition by using surface force apparatus, see Fig. 3. For stresses below the critical wall shear stress, the hydrophobic tails of the surfactant molecules remain interdigitated, the bilayer is responding elastically, and the stick condition is observed. Beyond this critical value, the surfactant molecules are disentangled and slip is observed. We relate the apparent concentration dependence on the critical pressure gradient to the surfactant coverage, which is known to vary slowly above the CMC with increasing surfactant bulk concentration [18].

This is corroborated by Fig. 4 where results from surface plasmon resonance (SPR) experiments are reported. In these experiments, surfactant solutions are flown past PVP coated gold surfaces. The gold surface plasmon resonance shifts with changes in refractive index distri-

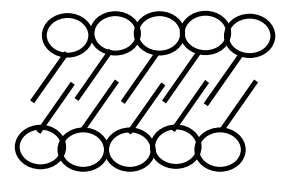

(a)

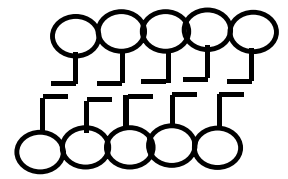

(b)
FIG. 3. Artist's impression of the response of the surfactant bilayer under an applied shear stress. Below a critical yield stress value, the layer responds elastically (a). Beyond this yield stress, the layers flow with respect to one another (b). At the critical yield stress, the interdigitated structure of the bilayer ruptures. bution close to the surface. Figure 4 clearly demonstrates that the structure of the surfactant bilayer changes with flow rate and hence with wall shear stress. Below surfactant concentration of $2 \mathrm{CMC}$, we do not observe a stickslip transition which is most likely due to the fact that in such flow conditions a uniform bilayer is not fully developed. Rather, surface concentration gradients leading to the Marangoni effect are expected to add to the surface stress. This is not taken into account here.

Another possible model considers two concentric phases flowing past one another where the outer sticks to the nanopore wall. In order to analyze this model, both the thickness and the viscosity of the surface layer must be known. A stick-slip transition will take place only if a Bingham-like fluid flow is assumed for the surface layer. Because of the larger number of parameters involved, we prefer the present model with only two experimentally accessible parameters.

To conclude, we experimentally showed that surfactant solution flow through nanopores suddenly increases intensity beyond a well-defined pressure gradient. This is interpreted as a changeover from fluid sticking to slipping at the wall. Such behavior is observed because of an adsorbed layer of surfactant at the solid-liquid interphase effectively behaving as a Bingham fluid [19]. As it appears from our analysis, the bigger the pore radius the smaller the critical wall shear stress but the smaller the slip concentration relative to the total flow. Small pore sizes, where these effects would be noticeable, are found in capillary viscometers. When $\lambda \ll R$, results of this technique should be reliable. However, when $\lambda \sim R$, as will be frequently encountered with

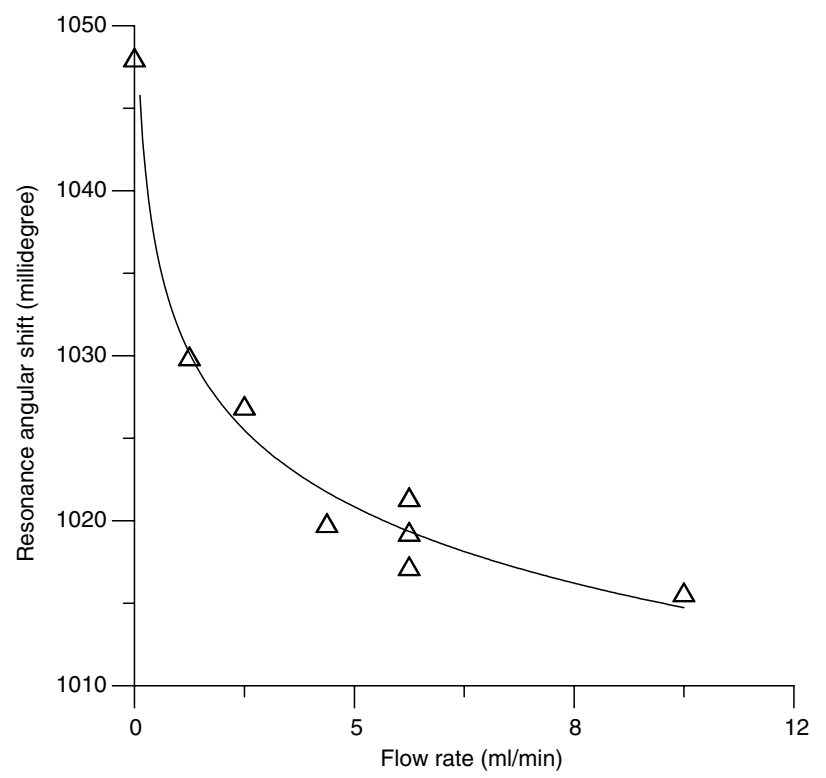

FIG. 4. SPR experiments conducted under various shear stresses exhibit flow dependence with surfactant solutions. The drawn line serves as guide to the eyes. 
complex fluids such as polymer solutions or polymer melts [11], the obtained viscosity readings should be corrected for slip.

The authors gratefully acknowledge G. Frens for constructive discussions.

[1] H. Lamb, Hydrodynamics (Dover Publications, New York, 1976), 6th ed., p. 576.

[2] N. V. Churaev, V. D. Sobolev, and A. N. Somov, J. Colloid Interface Sci. 97, 574 (1984).

[3] I. L. Singer, J. Vac. Sci. Technol. A 12, 2605 (1994).

[4] E. Bonaccurso, M. Kappl, and H. J. Butt, Phys. Rev. Lett. 88, 076103 (2002).

[5] Y. Zhu and S. Grannick, Phys. Rev. Lett. 87, 096105 (2001).

[6] Y. Zhu and S. Grannick, Langmuir 18, 1058 (2002).

[7] A. M. D. E. Timmerman, Ph.D. thesis, TU Delft, 2002.

[8] A. Patist, S. G. Oh, R. Leung, and D. O. Shah, Colloids Surf. A 176, 3 (2001).
[9] D. J. M. Bergink-Martens and G. Frens, Tensile Surfactants Deterg. 34, 263 (1997).

[10] A. M. Albano, D. Bedeaux, and P. Mazur, Physica (Amsterdam) 80A, 89 (1975).

[11] P. G. de Gennes, Langmuir 18, 3413 (2002).

[12] H. Yoshizawa, Y. L. Chen, and J. Israelachvili, J. Phys. Chem. 97, 4128 (1993).

[13] H. Yoshizawa and J. Israelachvili, J. Phys. Chem. 97, 11300 (1993).

[14] S. Levine, J. R. Marriott, G. Neale, and N. Epstein, J. Colloid Interface Sci. 52, 136 (1975).

[15] N. Fadnavis and J. B. F. N. Engberts, J. Am. Chem. Soc. 106, 2636 (1984).

[16] E. Minatti, D.P. Norwood, and W. F. Reed, Macromolecules 31, 2966 (1998).

[17] P. Richetti, C. Drummond, J. Israelachvili, M. In, and R. Zana, Europhys. Lett. 55, 653 (2001).

[18] S. D. Christian and J. F. Scamehorn, Solubilization in Surfactant Aggregates (M. Dekker, New York, 1995), 1st ed., p. 281.

[19] W. L. McCabe, J.C. Smith, and P. Harriot, Unit Operations of Chemical Engineering (McGraw-Hill, New York, 1993), 5th ed., pp. 89-91. 